Oct 21st, 2016

\title{
Intensity-based Choroidal Registration Using Regularized Block Matching
}

Tiziano Ronchetti

University of Basel

Peter Maloca

University of Basel

Christoph Meier

Bern University of Applied Sciences

Selim Orgül

University of Basel

Christoph Jud

University of Basel

See next page for additional authors

Follow this and additional works at: http://ir.uiowa.edu/omia

Part of the Ophthalmology Commons

\section{Recommended Citation}

Ronchetti, Tiziano; Maloca, Peter; Meier, Christoph; Orgül, Selim; Jud, Christoph; Hasler, Pascal; Považay, Boris; and Cattin, Philippe C.. Intensity-based Choroidal Registration Using Regularized Block Matching. In: Chen X, Garvin MK, Liu J, Trucco E, Xu Y editors. Proceedings of the Ophthalmic Medical Image Analysis Third International Workshop, OMIA 2016, Held in Conjunction with MICCAI 2016, Athens, Greece, October 21, 2016.33-40. Available from https://doi.org/10.17077/omia.1044

Rights

Copyright (C) 2016 the authors

Hosted by Iowa Research Online. For more information please contact: lib-ir@uiowa.edu. 
Presenter Information

Tiziano Ronchetti, Peter Maloca, Christoph Meier, Selim Orgül, Christoph Jud, Pascal Hasler, Boris Považay, and Philippe C. Cattin 


\title{
Intensity-based Choroidal Registration Using Regularized Block Matching
}

\author{
Tiziano Ronchetti ${ }^{1,2}$, Peter Maloca ${ }^{3}$, Christoph Meier $^{2}$, Selim Orgül ${ }^{3}$, \\ Christoph Jud ${ }^{1}$, Pascal Hasler ${ }^{3}$, Boris Považay ${ }^{2}$, and Philippe C. Cattin ${ }^{1}$ \\ ${ }^{1}$ Department of Biomedical Engineering (DBE), University of Basel, ${ }^{2}$ Institute for \\ Human Centered Engineering (HuCE)-optoLab, Bern University of Applied Sciences, \\ ${ }^{3}$ OCTlab, Department of Ophthalmology, University of Basel \\ Tiziano.Ronchetti@unibas.ch
}

\begin{abstract}
Detecting and monitoring changes in the human choroid play a crucial role in treating ocular diseases such as myopia. However, reliable segmentation of optical coherence tomography (OCT) images at the choroid-sclera interface (CSI) is notoriously difficult due to poor contrast, signal loss and OCT artefacts. In this paper we present blockwise registration of successive scans to improve stability also during complete loss of the CSI-signal. First, we formulated the problem as minimization of a regularized energy functional. Then, we tested our automated method for piecewise Intensity-based Choroidal rigid Registration using regularized block matching (ICR) on 20 OCT 3D-volume scan-rescan data set pairs. Finally, we used these data set pairs to determine the precision of our method, while the accuracy was determined by comparing our results with those using manually annotated scans.
\end{abstract}

Keywords: choroid-sclera, retinal layers, regularized block matching

\section{Introduction}

Myopia is a disease manifested by a disproportional growth of the eye bulb leading to a reduction and loss of visual function. It is widely diagnosed in Asian urban regions and also strongly increases in western countries. It starts to develop at school age leading to $80 \%$ incidence among teenagers. The strongly varying thickness of the choroid, the blood-filled 50 to $300 \mu \mathrm{m}$ thick layer underneath the retina, encapsulated by the rigid sclera, is correlated to not only myopia, but also to various other ocular diseases like diabetic retinopathy, intraocular tumors and macular degeneration [6]. This simple indicator is defined as the distance between the Choriocapillaris-Bruch's membrane-Retinal pigment epithelium complex (CBR) and the Choroid-Sclera Interface (CSI, see Fig. 1c). Choroidal maps (see Fig. 1b) visualize the individually and locally strongly varying thickness distribution across the area of the central choroid and can aid to extract essential clinical information. Likewise, the thickness of the retina, i.e. the distance between the Inner Limiting Membrane (ILM) and the CBR, is visualized by retinal maps (see Fig. 1a). The segmentation of the CBR is relatively

X. Chen, M. K. Garvin, J. Liu, E. Trucco, Y. Xu (Eds.): OMIA 2016, Held in Conjunction with MICCAI 2016, Athens, Greece, Iowa Research Online, pp. 33-40, 2016. Available from: http://ir.uiowa.edu/omia/2016_Proceedings/2016/ 
simple (e.g. using automatic segmentation methods based on graph theory [3]) because of the strong and characteristic signal of the retinal images in Optical Coherence Tomography (OCT). It has become the main contact-free, non-invasive, high-resolution imaging method that enables the detection of $\mu \mathrm{m}$ changes in the human retina and the choroid [7]. Segmenting the CSI, however, is very difficult due to the significantly lower image contrast, an increase of shadowing artefacts and unpredictable shape variations of the choroid [6]. Several studies (e.g.

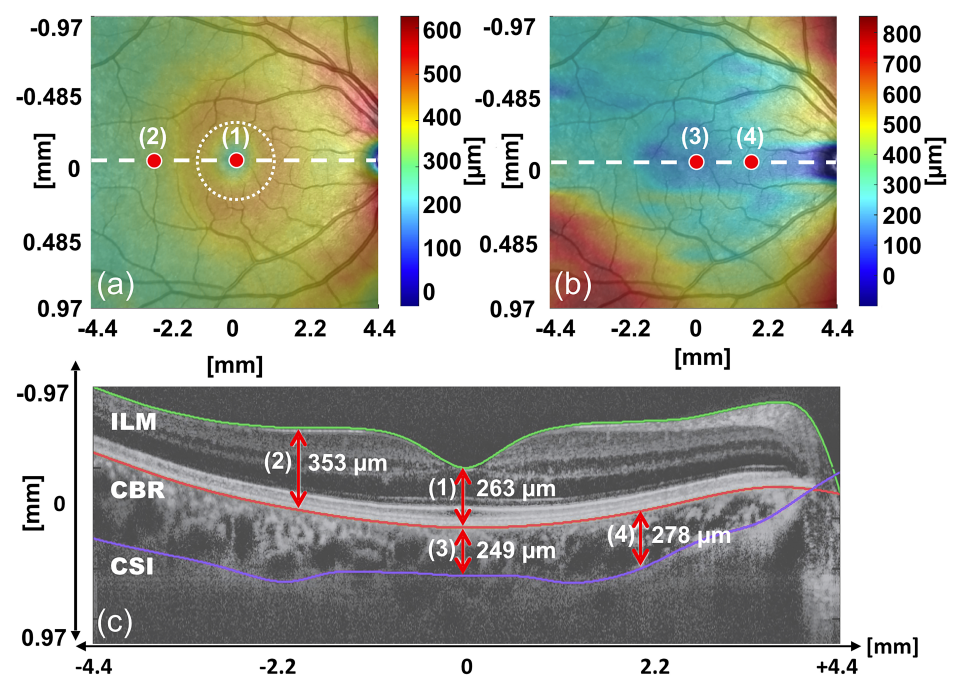

Fig. 1: (a) Retinal (ILM-CBR) and (b) choroidal (CBR-CSI) thickness maps calculated with ICR overlaid color-coded on fundus image. (c) OCT B-scan with segmented ILM, CBR and CSI layers.

$[3],[5],[9])$ use single frame segmentation, which works well in cases with good signal quality and smooth interfaces. However, this becomes difficult with less compliant subjects or in longitudinal clinical studies where successive imaging sessions can strongly vary in signal quality, leading to strong variations in image and segmentation quality [5]. Thus, reliable quantification for detection of subtle changes of choroidal thickness across a long sequence of sessions is error prone and insufficient for precise monitoring.

Instead of segmenting each tomogram individually, we suggest using image registration of image sequences obtained during a longitudinal study, utilizing the a-priori topological knowledge from previous acquisitions.

Conventional registration methods (see review article [10]) will fail when registering such OCT-data of the human eye (see Sec. 4). This is caused by the interleaved nature of the eye consisting of the soft choroid and surrounding more rigid tissues, the sclera and the CBR. It is therefore important, that sclera and CBR are treated as a rigid body during registration. Although a non-rigid 


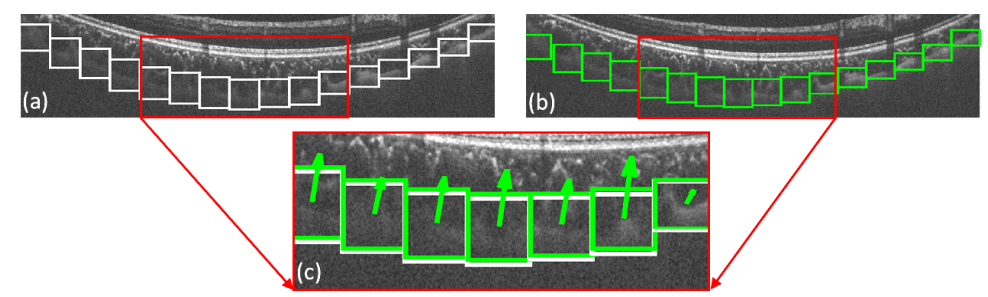

Fig. 2: Reference (a) and registered image (b) with corresponding blocks around the approximately determined CSI layer. Image (c) shows the result of the regularized block matching represented by the optimal position (green blocks) and the displacement field (green arrows) required for compensation.

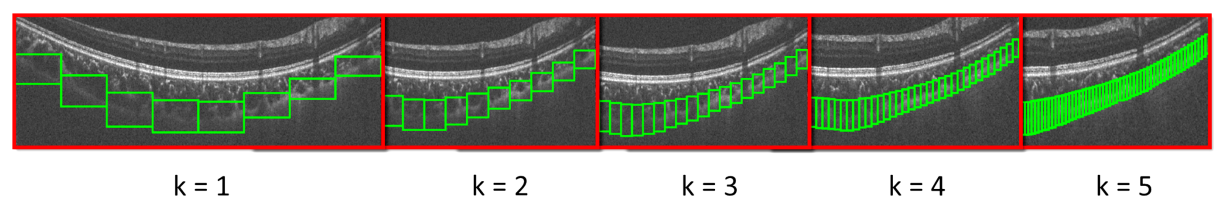

Fig. 3: Refinement of regularized block matching using different levels of subdivisions between 8 blocks $(\mathrm{k}=1)$ and 128 blocks $(\mathrm{k}=5)$.

registration procedure is imposed by the physical characteristics of the choroid, a piecewise rigid strategy [2] seems to be the most suitable and reliable approach to accurately model the deformation of the choroid. It allows us to decompose the global non-rigid matching problem of the choroid into numerous local rigid registrations of the individual subregions. The results are then embodied into a dense global non-rigid deformation field built in such a way that it elastically deforms the soft choroid and preserves the rigid characteristics of the surrounding sclera and CBR.

Our ICR method is based on the pre-segmentation of the CBR and CSI, which is tracked via registration in successive scans to quantify its growth. On the basis of the accurately segmented CBR, we approximate the position and shape of the CSI. Utilizing the rigid CBR as a reference line, we conduct hierarchical regularized block-matching [4] registration of the CSI (see Fig. 2 and 3). As the images were aligned at the rigid CBR, the displacement corresponds to either an increase or decrease of the CSI interface (see Fig. 2). This allows us to determine the displacement field in the vicinity of the CSI layer and use the outcome as a basis to quantify any choroidal growth.

\section{Material \& Method}

In this section we first introduce the image acquisition process and then we explain the processing pipeline in more detail. The OCT systems used were enhanced Spectralis OCT (Heidelberg Engineering) prototypes operating simultaneously at $800 \mathrm{~nm}$ and $1075 \mathrm{~nm}$ wavelength for obtaining different tissue contrast 
and penetration. The development of these dual wavelength SD-OCT system, called Hydra Spectralis, was conducted at the University of Applied Sciences in Biel, Switzerland. One of the systems is installed in the Centre for Myopia Research in Hong Kong, China, to obtain clinical data for multiple cross-sectional and longitudinal clinical studies in children and teenagers. We used 20 OCT 3D-volume data set pairs acquired as scan-rescan of 20 different Asian children between 8 and 13 years old. Ten children had their left eye and ten others had their right eye measured twice within a span of a few minutes. Each volume-

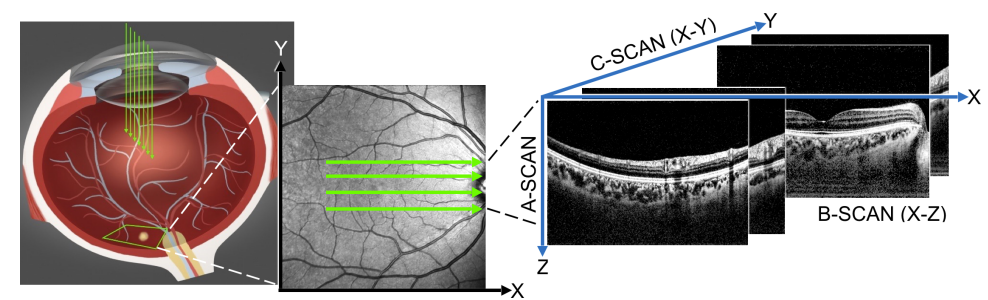

Fig. 4: The OCT scan acquisition system Hydra Spectralis.

stack consists of $25 \mathrm{~B}$-scans at a lateral resolution in nasal-temporal/x-direction (see Fig. 4) was set to 768 depth-scans per frame, acquired at 20 frames/second. The axial resolution (in depth/z-direction) in the high speed acquisition mode is $11.46 \mu \mathrm{m} /$ pixel respectively $4.70 \mu \mathrm{m} /$ pixel. The scanned area is $30^{\circ} \times 20^{\circ}$ in $x$ and $y$ (including the optical nerve). Reproducibility of the scan locations is maintained by a scanning laser ophthalmoscope (SLO)-based eye-tracking system that independently raster scans the eye at $\mu \mathrm{m}$-precision and laterally repositions the OCT-beams to the target area, thereby compensating involuntary eye movement by stabilizing the $z$-position with a sturdy head-rest.

\subsection{Preprocessing}

Acquisition: A stack of up to 100 B-scans is acquired at the same position; frames are checked versus the SLO position and converted to images.

Stack registration: The acquired number of B-scans of each location and session are registered based on a similarity transformation (translation, rotation, scaling). The registration method is extended by a three-level image pyramid for multi-resolution image registration. The optimizer used for this task is a Regular Step Gradient Descent algorithm. Images that do not pass a pre-set error-level of 0.2 based on the normalized cross correlation are rejected. The registration result is a 16-bit grayscale image.

Contrast enhancement and noise reduction: To enhance the contrast, histogram equalization is applied to the original grayscale OCT image. To remove noise and locally improve the signal-to-noise ratio, Wiener filtering is slice-wise used. The cohesion of regions is improved with slice-wise median filtering. The averaged B-scans of multiple imaging sessions are combined to a new stack. 
Rigid registration of multi-session stack: Here we roughly align pairs of corresponding B-scans from the reference and floating stack images at the CBR level. To speed up this registration process we crop the images to remove the uninteresting areas with a lot of noise above the ILM and beneath the choroid. Segmentation of ILM and CBR: Using the on graph search based algorithm presented in [3], we accurately segment the ILM and the CBR. Further on the segmented CBR becomes the shape-reference for the CSI.

\subsection{Piecewise rigid registration using regularized block matching}

Let $\Omega_{j} \subset \mathbb{R}^{2}$ be the $j^{t h}$ B-scans of size $m \times n\left[\right.$ pixel $\left.^{2}\right]$ in a volume stack $\Omega=$ $\bigcup_{j=1}^{S} \Omega_{j} \subset \mathbb{R}^{3}$. We consider a reference $I_{R}$ and a template image $I_{T}: \Omega \rightarrow \mathbb{R}$ mapping $\Omega$ to the corresponding intensities. In this context, we consider image registration as a regularized minimization problem for the energy functional $\mathcal{J}$,

$$
\min _{u} \mathcal{J}[u], \quad \mathcal{J}[u]=\mathcal{D}\left[I_{R}, I_{T}, u\right]+\lambda \mathcal{R}[u]+\mu \mathcal{S}[u] .
$$

$\mathcal{D}$ denotes a distance measure that quantifies the similarity between reference $I_{R}$ and transformed image $I_{T}(p+u(p))$. $\mathcal{R}$ and $\mathcal{S}$ are regularization terms with the corresponding balancing parameters $\lambda$ and $\mu$. They ensure certain properties of the transformation, which we will explain next.

In each B-scan we divide the interval $[0, n]$ into $N$ equidistant cells of width $\omega=\frac{n}{N}$ and cell centers $x_{i}=(2 i-1) \frac{\omega}{2}, i=1, \ldots, N$. Let $d_{i}$ be the distance between the segmented CBR and the roughly determined CSI at each location $x_{i}$, let $k_{a}$ and $k_{u}$ be two constants. We describe with $k_{a} \cdot d_{i}$ the block part above the CSI and with $k_{u} \cdot d_{i}$ the block part below. Thus, we obtain in each B-scan $\Omega_{j}$ non-overlapping rectangular blocks $\left\{\mathcal{B}_{i}^{j}\right\}_{i=1}^{N}$ with constant width $\omega$, variable height $h_{i}=\left(k_{a}+k_{u}\right) \cdot d_{i}$ and center points $p_{i}$.

The aim is to find a $\operatorname{set} \mathcal{U}=\left\{u_{i}^{j}\right\}$ of blockwise constant transformations $u_{i}^{j} \in$ $\mathbb{R}^{3}$ such that $I_{T}\left(p+u_{i}^{j}\right) \approx I_{R}(p)$ for all $p \in \mathcal{B}_{i}^{j}$. $\mathcal{U}$ represents the displacement field between corresponding scans from the same individual.

At the first resolution level $k=1$ we initialize the block matching with 8 blocks. Applying a hierarchical strategy [1], each time we obtain $N=16,32, \ldots, 128$ blocks for every following level $k=2, \ldots, 5$ (see Fig. 3). Using piecewise intensitybased local rigid registration, we attempt to obtain the maximum correlation by matching blocks of the pre-determined CSI layer from the pre-registered image with the ones in the corresponding, slightly bigger search area $\hat{\mathcal{B}}_{i}^{j} \supset \mathcal{B}_{i}^{j}$.

We define the similarity measure

$$
\mathcal{D}\left[I_{R}, I_{T}, u_{i}^{j}\right]=\sum_{j=1}^{S} \sum_{i=1}^{N} \int_{p \in \mathcal{B}_{i}^{j}} \mathcal{G}_{i}(p) \mathcal{L}\left(I_{T}\left(p+u_{i}^{j}\right), I_{R}(p)\right) d p,
$$

using as loss function $\mathcal{L}$ the $L_{2}$ norm $=\|\cdot\|^{2}$ :

$$
\mathcal{L}\left(I_{T}\left(p+u_{i}^{j}\right), I_{R}(p)\right)=\left\|I_{T}\left(p+u_{i}^{j}\right)-I_{R}(p)\right\|^{2},
$$




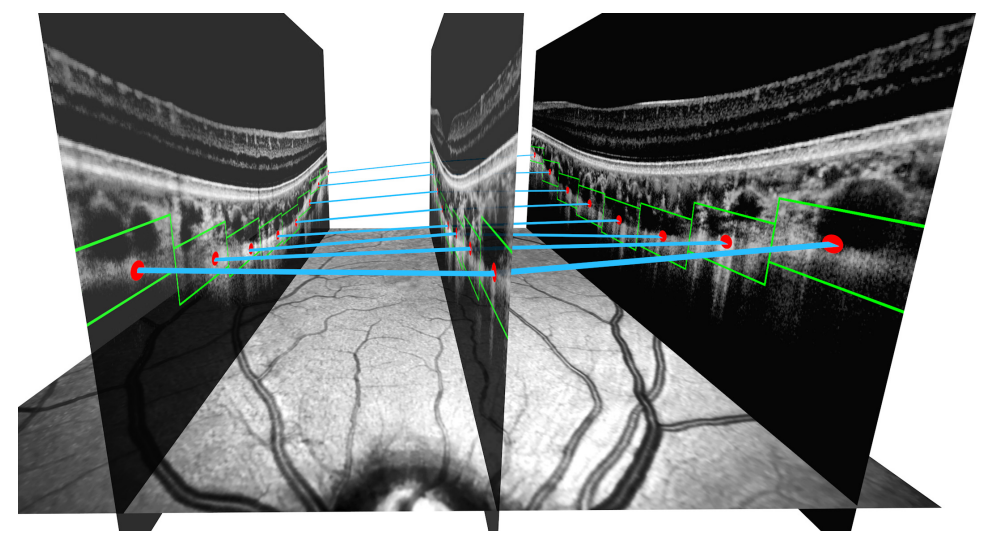

Fig. 5: With the term $S\left[u_{i}^{j}\right]$ we regularize in the scan depth across B-scans (note that the distance between the slices has been increased for better visualization). Blue lines indicate correlations between sites.

where $p$ is the point position in block $\mathcal{B}_{i}^{j} \subset \Omega_{j}$ and

$$
\mathcal{G}_{i}(p): \Omega_{j} \rightarrow \mathbb{R}_{0}^{+}, \quad \mathcal{G}_{i}(p)= \begin{cases}e^{-\left\|p-\hat{p}_{i}\right\|^{2}} & \text { if } \hat{p}=p+u_{i}^{j} \in \hat{\mathcal{B}}_{i}^{j} \\ 0 & \text { otherwise }\end{cases}
$$

is a weighting function which penalizes matches far off the target, where $\hat{p}_{i}$ is the center point of the matched block. Furthermore, we calculate for each B-scan the optimal $2^{\text {nd }}$ order polynomial which approximates the CBR-layer to obtain $\mathcal{K}$, the average of all curvatures. We use $\mathcal{K}$ as lower boundary for the regularization term $\mathcal{R}$,

$$
\mathcal{R}\left[u_{i}^{j}\right]=\sum_{j=1}^{S} \sum_{i=1}^{N}\left(\left\|D_{x}^{2}\left[u_{i}^{j}\right]\right\|-\mathcal{K}\right)^{2}
$$

where $D_{x}^{2}$ is the $2^{\text {nd }}$ order difference operator in $x$-direction. We calculate $D_{x}^{2}$ by fitting a cubic spline through the centers of the blocks and calculating its $2^{\text {nd }}$ order derivative. $\mathcal{R}$ penalizes non-smooth solutions, to avoid extreme values of the curvature of the CSI layer. In this way we regularize the vertical displacements of the blocks within a B-scan in comparison to the blocks of the corresponding reference scan. With the term $\mathcal{S}$,

$$
\mathcal{S}\left[u_{i}^{j}\right]=\sum_{j=1}^{S} \sum_{i=1}^{N}\left\|D_{y}^{2}\left[u_{i}^{j}\right]\right\|_{,}^{2}
$$

we regularize in the depth of the volume stack $\Omega$ (in $y$-direction, see Fig. 5). We calculate $D_{y}^{2}$ similarly to $D_{x}^{2}$. With this additional regularization we take into account not only the adjacent blocks within the same B-scan, but also the corresponding blocks in the neighboring scans. 
Table 1: Mean $(\bar{x})$, standard deviation $(s)$ and median $(\tilde{x})$ of the 20 data set pairs (in $[\mu \mathrm{m}]$ ) for ICR and manual (man.) registration.

\begin{tabular}{c||cc|cc|cc|cc|cc|}
\multicolumn{1}{c||}{} & \multicolumn{2}{c|}{$\mathrm{k}=1$} & \multicolumn{2}{c|}{$\mathrm{k}=2$} & \multicolumn{2}{c|}{$\mathrm{k}=3$} & \multicolumn{2}{c|}{$\mathrm{k}=4$} & \multicolumn{2}{c|}{$\mathrm{k}=5$} \\
\hline Method & ICR & man. & ICR & man. & ICR & man. & ICR & man. & ICR & man. \\
\hline $\bar{x}$ & 3.52 & 6.03 & 3.62 & 7.16 & 3.92 & 8.64 & 4.15 & 10.44 & 6.81 & 15.96 \\
$s$ & 0.92 & 1.91 & 1.58 & 3.08 & 1.83 & 4.15 & 2.25 & 5.85 & 3.69 & 7.94 \\
$\tilde{x}$ & 3.13 & 5.86 & 3.43 & 6.03 & 3.20 & 7.39 & 3.39 & 9.31 & 6.06 & 17.29
\end{tabular}

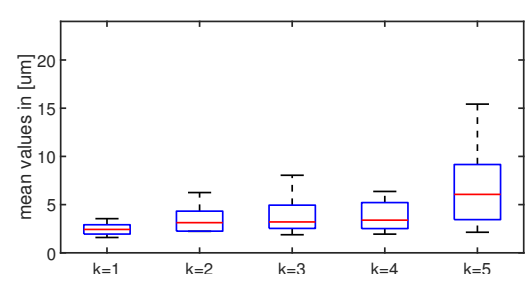

(a) Our method ICR

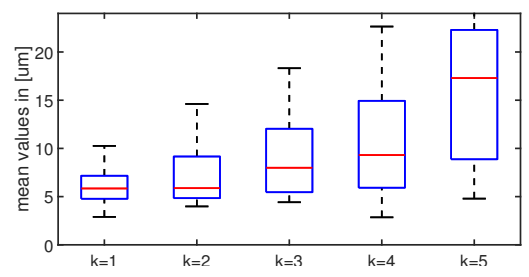

(b) Manual detection

Fig. 6: The automated (a) and the manual (b) detection of block displacements.

\section{Results}

We have tested our algorithm on the 20 data set pairs for each level $k$ and compared it to manual segmentation by a professional ophthalmologist. The results are shown in Tab. 1. Figure 6a depicts the mean values of all the block displacements in the template image during the regularized block matching for all 20 data set pairs. To get the optimal combination of precise results and a high spatial resolution, the best level of registration for our data set is $k=4$. This enables us to find choroidal changes at 64 different positions. Starting with $k=5$ a significant deterioration of the scan-rescan results can be observed. This is caused by the spatially high frequency noise of speckle that cannot be counteracted by a large structural signal in such a fine subdivision and leads to improper patch registration. Consequently, the results of the block matching algorithm become inaccurate. Figure $6 \mathrm{~b}$ shows the mean values of all the block displacements after manual segmentation of the CBR and CSI, followed by manual block building and matching (the blocks have been located by an expert as described in Sec. 2.2). A comparison of the accuracy of our method ICR and the manual detection has shown that, with increasing spatial resolution, our method still recognizes minute changes $<5 \mu \mathrm{m}$ up to the level $k=4$, which could not be attained by an ophthalmologist, even at a higher resolution.

\section{Discussion \& Outlook}

As mentioned in Sec. 1 the application of general 3D registration algorithms is in this case not useful, i.e. even the use of the smoothing parameter sigma 
by Demons registration algorithm [8] does not allow a satisfactory registration. With a small value of sigma the template matches the image of reference, but deformations are produced. By increasing the value of sigma, the deformations are reduced, but the images can no longer be aligned. The total time needed by

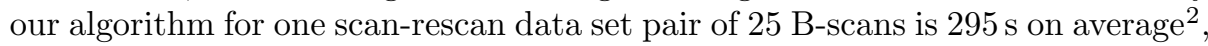
which already makes it usable in clinical routine. We believe that an improvement in terms of computation time can be achieved with parallelization in MATLAB, followed by implementing the method in $\mathrm{C}++$. We intend to apply our method on test persons over an extended period of time and later also on patients with eye diseases. In addition, we plan to investigate the connection between the shape of the eye (curvature of the sclera) and myopia. We also consider the possibility to apply our algorithm in other fields (e.g. OCT-dermatology, ultrasound imaging).

\section{References}

1. Andronache, A., von Siebenthal, M., Székely, G., Cattin, P.: Non-rigid registration of multi-modal images using both mutual information and cross-correlation. Medical image analysis 12(1), 3-15 (2008)

2. Čech, P., Andronache, A., Wang, L., Székely, G., Cattin, P.: Piecewise rigid multimodal spine registration. In: Bildverarbeitung für die Medizin 2006, pp. 211-215. Springer (2006)

3. Chiu, S.J., Li, X.T., Nicholas, P., Toth, C.A., Izatt, J.A., Farsiu, S.: Automatic segmentation of seven retinal layers in SDOCT images congruent with expert manual segmentation. Optics express 18(18), 19413-19428 (2010)

4. De Haan, G., Biezen, P.W., Huijgen, H., Ojo, O.A.: True-motion estimation with 3D recursive search block matching. Circuits and Systems for Video Technology, IEEE Transactions on 3(5), 368-379 (1993)

5. Kajić, V., Esmaeelpour, M., Považay, B., Marshall, D., Rosin, P.L., Drexler, W.: Automated choroidal segmentation of $1060 \mathrm{~nm}$ OCT in healthy and pathologic eyes using a statistical model. Biomedical optics express 3(1), 86-103 (2012)

6. Morgan, I.G., Ohno-Matsui, K., Saw, S.M.: Myopia. The Lancet 379(9827), 17391748 (2012)

7. Považay, B., Hermann, B., Unterhuber, A., Hofer, B., et al.: Three-dimensional optical coherence tomography at $1050 \mathrm{~nm}$ versus $800 \mathrm{~nm}$ in retinal pathologies: enhanced performance and choroidal penetration in cataract patients. Journal of biomedical optics 12(4), 041211-041211 (2007)

8. Thirion, J.P.: Image matching as a diffusion process: an analogy with maxwell's demons. Medical image analysis 2(3), 243-260 (1998)

9. Tian, J., Marziliano, P., Baskaran, M., Tun, T.A., Aung, T.: Automatic segmentation of the choroid in enhanced depth imaging optical coherence tomography images. Biomedical optics express 4(3), 397-411 (2013)

10. Zitova, B., Flusser, J.: Image registration methods: a survey. Image and vision computing 21(11), 977-1000 (2003)

\footnotetext{
${ }^{2}$ Microsoft Windows 7 64-bit, MATLAB, R2015b, from MathWorks, Inc. (Natick, MA; US), $2.90 \mathrm{GHz}$ Intel Core i7-3520M processor with 8 GB RAM
} 